\title{
Análisis de la calidad de vida en pacientes en hemodiálisis ambulatoria y su relación con el nivel de dependencia
}

\author{
Tamara Albañil Frías, María del Carmen Ramírez Moreno, Rodolfo Crespo Montero \\ Centro Periférico de Diálisis Perpetuo Socorro. U.G.C. Nefrología. Hospital Reina Sofía de Córdoba. Facultad de \\ Medicina y Enfermería de Córdoba. Fundación Nefrológica de Córdoba
}

\section{Resumen}

Introducción: Se ha asociado una baja calidad de vida relacionada con la salud (CVRS) en pacientes en hemodiálisis (HD) con ser mujer, mayor edad, estado civil, situación laboral, etc. Sin embargo, no existen muchos estudios que profundicen sobre la interacción entre deterioro cognitivo, calidad de vida, ancianidad y HD, motivo por el cual nos planteamos la realización de este estudio.

Objetivo: Analizar la CVRS de los pacientes con insuficiencia renal crónica sometidos a tratamiento sustitutivo con HD ambulatoria y su relación con el nivel de dependencia.

Pacientes y Métodos: Se evaluó la CVRS a 43 pacientes en HD del centro periférico de diálisis del HURS de Córdoba, mediante el cuestionario de salud SF-36. Para el análisis de dependencia se utilizó la escala de Karnofsky. También se recogieron variables sociodemográficas y el índice de comorbilidad de Charlson (ICC).

Resultados: Con respecto a la CVRS se encontró: Función física 49.6 \pm 30 , Salud general $40.4 \pm 22$, Rol físico 50.5 \pm 34.4 , Rol emocional 81.7 \pm 28.3 , Dolor corporal 55.6 \pm 31.9 , Vitalidad 51.4 \pm 31.5 , Función social $58.43 \pm 36$, Salud mental $69.6 \pm 22.8$. Respecto a la escala de Karnosfky fue de $76.5 \pm 24.7$ puntos (el $55.8 \%$ de los pacientes eran capaces de realizar actividades normales, el $34.9 \%$ eran incapaces de trabajar y el $9.4 \%$ eran incapaces de autocuidarse). Se encontró correlación de la edad con la escala de Karnofsky $(r=-0.58, p<0.01)$ y con el ICC $(r=0.81, p<0.01)$; del ICC con Karnofsky $(r=-0.54, p<0.01)$, la dimensión salud mental $(r=0.36, p<0.05)$, la dimensión función física $(r=-0.43, p<0.01)$ y la dimensión función social $(r=-0.31, p<0.05)$.

\section{Correspondencia:}

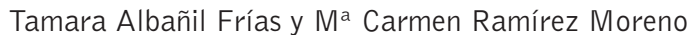
Centro Periférico de Diálisis

C/ Virgen del Perpetuo Socorro, 12

14004. Córdoba

E-mail:n02alfrt@uco.es
Conclusiones: Estos resultados ponen de manifiesto que los pacientes estudiados presentan peor CVRS, con bajas puntuaciones en todas las dimensiones del SF-36 en comparación con los valores de referencia de la población general española. Entre ellas, la dimensión rol físico es la más afectada, seguida de la función social y función física, respectivamente; y las menos afectadas son las dimensiones salud mental y rol emocional. Esta peor CVRS se asocia con mayor edad, comorbilidad y dependencia.

PALABRAS CLAVE

- CALIDAD DE VIDA

- HEMODIÁLISIS

- DEPENDENCIA

- COMORBILIDAD

Analysis of the quality of life in ambulatory hemodialysis patients and its relationship to the degree of dependence

\section{Abstracts}

Introduction. A low quality of life has been related to health (HRQL) in patients on hemodialysis (HD), female sex, adulthood, marital status, employment situation, etc. However, there are not many studies that go into the interaction among cognitive decay, quality of life, dotage and HD in depth. This is the reason why we considered to carry on this study.

Objetive: To anlyse the HRQL for patients suffering renal failure and receiving substitutional treatment with outpatient HD and its relation to the dependence level.

Patients and Methods: It was conducted the SF36 health survey to assess the HRQL of forty-three patients in HD from the dialysis outlying centre of HURS in Córdoba. For the dependence analysis it was used the Karnofsky scale. It was also included sociodemographic variables and Charlson Comorbidity Index (CCI). 
Results: In relation to the HRQL it was found: Physical function 49.6 \pm 30 , General health $40.4 \pm 22$, Physical role $50.5 \pm 34.4$, Emotional role $81.7 \pm 28.3$, Body pain $55.6 \pm 31.9$, Vitality $51.4 \pm 31.5$, Social function $58.43 \pm 36$, Mental health $69.6 \pm 22.8$. The Karnosfky scale score was $76.5 \pm 24.7(55.8 \%$ of patients were able to do normal activities, $34.9 \%$ were able to work and $9.4 \%$ were able to take care of themselves). It was found correlation between age and Karnofsky scale $(r=-0.58, p<0.01)$ and with the CCI $(r=0.81, p<0.01)$; correlation between the CCI and Karnofsky $(r=-0.54, p<0.01)$, mental health dimension ( $r=0.36, p<0.05)$, physical function dimension $(r=-0.43, p<0.01)$ and social function dimension ( $r=-$ $0.31, p<0.05)$.

Conclusions: These results show that the patients tested have worse HRQL, with low score in all SF36 dimensions comparing to the reference values of Spanish general population. Among them, the physical role dimension is the most affected, followed by the social and physical function dimensions respectively; and the least affected are the mental health and emotional role dimensions. This worse HRQL is associated to higher age, comorbidity and dependence.

\section{KEYWORDS}

- QUALITY OF LIFE
- HEMODIALYSIS
- DEPENDENCE
- COMORBIDITY

\section{Introducción}

En los últimos años, hemos asistido a un envejecimiento de la población provocado, en parte, por un aumento de la esperanza de vida. Los 34,8 años de vida media o esperanza de vida al nacer en 1900 se han convertido en 81,6 años en 2009 . Además, con el paso del tiempo se irá agudizando el "envejecimiento del envejecimiento", característica demográfica que va a experimentar el colectivo de 80 y más años, cuyo ritmo de crecimiento superará al del grupo de población mayor de 65 años en su conjunto ${ }^{1}$. Parte del aumento de la esperanza de vida es atribuible a la importante disminución de la mortalidad en las edades más tempranas de la vida, disminución que se ha conseguido, básicamente, gracias a la reducción de la mortalidad por enfermedades infecciosas y parasitarias. Junto a la mayor duración de la vida media de la población, se ha producido un cambio en la estructura de la morbilidad con una importancia proporcionalmente mayor de las enfermedades crónicas y discapacitantes, au- mentando su incidencia y prevalencia a medida que la población envejece; convirtiéndose en un problema de gran relevancia e impacto para la Salud pública a nivel mundial ${ }^{2}$. Por lo general, las enfermedades diagnosticadas en los adultos mayores no son curables $y_{\text {, }}$ si no se tratan adecuada y oportunamente, tienden a provocar complicaciones y secuelas que dificultan la independencia y la autonomía de las personas ${ }^{3}$.

La HD ha permitido y permite vivir a un número importante de personas en el mundo con IRT, no obstante, no resuelve todos los problemas derivados de la enfermedad: desnutrición debida a la eliminación de nutrientes, insomnio, fatiga, pérdida de movilidad, cansancio, palidez, etc, además de la dependencia vital que crea en el paciente, pues necesita una máquina para vivir, teniendo que asistir al menos tres veces por semana al centro asistencial y permanecer allí de tres a cuatro horas, dependiendo de las necesidades de cada paciente ${ }^{4,5}$.

Por otro lado, y debido fundamentalmente a la mayor supervivencia del paciente en diálisis, estos presentan mayor comorbilidad asociada. En efecto, estos pacientes van a presentar múltiples patologías ligadas a la IRT, como son diabetes, hipertensión arterial, cardiopatía isquémica, hipercolesterolemia, vasculopatía periférica. Asimismo, la mayor accesibilidad de la terapia dialítica, el envejecimiento y el mayor tiempo de permanencia en HD también han contribuido a la presencia habitual de dolor en estos pacientes, en especial dolor crónico ${ }^{6}$. Además de estos síntomas físicos, el paciente en HD va a experimentar grandes cambios en su vida personal, derivados de las restricciones que impone vivir dependiente de una máquina, y las implicaciones que esto va a tener en todos los ámbitos de su vida. Afrontar una enfermedad crónica y progresiva, altamente demandante, cuyo tratamiento es invasivo y continuado, produce de manera permanente importantes cambios de vida en los ámbitos físico, psicológico y social tanto para el paciente y como para su familia?.

Por tanto, si tenemos en cuenta todos los determinantes anteriormente citados: edad, comorbilidad asociada, limitaciones físicas, es frecuente que los pacientes en HD presenten algún grado de discapacidad o dependencia, lo cual los hace especialmente dependientes del sistema formal e informal de cuidados para satisfacer su necesidades. La dependencia puede entenderse como el resultado de un proceso que se inicia con la aparición de un déficit en el funcionamiento corporal como consecuencia de una enfermedad o accidente. Este déficit comporta una limitación en la actividad 
para el individuo. Cuando esta limitación no puede compensarse mediante la adaptación del entorno, provoca una restricción en la participación que se concreta en la dependencia de la ayuda de otras personas para realizar las actividades de la vida cotidiana ${ }^{8,9}$; asociándose esta dependencia a morbimortalidad, siendo un predictor más de supervivencia ${ }^{10}$.

Además de las características de la IRT y su tratamiento, la incertidumbre con respecto al futuro, la dependencia a la máquina de diálisis y al personal sanitario, las limitaciones en la dieta, los cambios en las relaciones sociales y familiares y en el aspecto físico y la situación laboral, entre otros, explican la aparición de trastornos emocionales y cambios en la calidad de vida percibida $^{11,12}$.

En cuanto a las variables estudiadas, son muchos los trabajos que plantean las variables que influyen sobre la calidad de vida en este tipo de pacientes, unos con influencia positiva y otros con influencia negativa: a) sociodemográficas: mayor edad, sexo femenino, nivel educacional, nivel socioeconómico, estado civil, comorbilidad asociada, etc.; b) psicológicas: depresión, ansiedad, incertidumbre sobre el futuro, etc. ${ }^{13}$, encontrando en ocasiones contradicciones entre unos trabajos y otros. Se ha asociado una baja CVRS en pacientes en HD con: ser mujer, una edad mayor, el estado civil, la situación laboral, etc. Sin embargo, no existen muchos estudios que profundicen sobre la interacción entre deterioro cognitivo, calidad de vida, ancianidad y hemodiálisis, un problema cuya prevalencia aumenta con la edad y entre cuyos factores etiopatogénicos se baraja la patología vascular. Ambas características son cada vez más frecuentes en la población en diálisis, la cual ha variado considerablemente en los últimos años, de manera que ahora la edad media de inicio de hemodiálisis es sensiblemente mayor, así como la comorbilidad asociada ${ }^{14}$.

Por otro lado, las variables psicológicas que han mostrado ejercer efecto sobre la calidad de vida son la depresión, la ansiedad, la incertidumbre acerca del futuro y el reconocimiento de la pérdida de calidad de vida, lo cual tiene importantes repercusiones sobre el bienestar de los pacientes y se ha asociado con una mayor mortalidad. Otras reacciones frecuentes en los pacientes renales dializados que afectan su calidad de vida son la hostilidad, la ira, la dependencia emocional y psicológica derivada de la dependencia del tratamiento sustitutivo ${ }^{15}$.

Por todo ello, y con el propósito general de conocer la CVRS de los pacientes en HD nos planteamos el presente estudio.

\section{Objetivos}

El objetivo del presente estudio fue analizar la CVRS de los pacientes en HD y su relación con el nivel de dependencia.

\section{Pacientes y métodos}

\section{Ámbito del estudio}

Este estudio se ha realizado en el Centro Periférico de Diálisis del Servicio de Nefrología del Hospital Universitario Reina Sofía de Córdoba.

\section{Población y muestra}

La muestra fue intencional y abarcó, inicialmente, a los 59 pacientes con IRT en tratamiento con HD en el Centro Periférico de Diálisis de la UGC Nefrología de Córdoba.

Se consideraron los siguientes criterios de exclusión:

a) Pacientes que no aceptaran de forma voluntaria participar en el estudio. Se explicó a cada paciente el objetivo del estudio y se solicitó el consentimiento verbal a cada uno de ellos para poder participar en el mismo.

b) Edad inferior a 18 años.

c) Pacientes que registran menos de tres meses en tratamiento con HD y que por las características del tratamiento no se encontraban estabilizados.

d) Situación clínica grave, trastorno psiquiátrico o cognitivo que incapacite al paciente para responder al cuestionario.

e) Lengua materna distinta al español. La versión española no ha sido validada para estos casos.

f) Sufrir ingreso hospitalario en las cuatro semanas previas a la recogida de datos, ya que muchas preguntas se refieren a cómo se sintió el paciente durante este periodo de tiempo.

Según estos criterios, del total de pacientes $(n=59)$, se excluyen 15 pacientes: 2 por deterioro cognitivo, 1 por idioma extranjero, 12 porque no dieron el consentimiento para participar y 1 por llevar menos de tres meses en tratamiento con HD. Finalmente, la muestra estuvo compuesta por 43 pacientes.

\section{Diseño del estudio}

Se realizó un estudio de diseño observacional analítico.

\section{Variables}

Las variables demográficas estudiadas fueron: edad, sexo, estado civil, tiempo en diálisis, profesión, tiempo 
que tarda en llegar al centro de diálisis, transporte utilizado, acceso vascular, situación laboral y composición del hogar.

Variables de resultado: calidad de vida, dependencia y comorbilidad.

\section{Instrumentos de medida}

Para la clasificación objetiva de las limitaciones funcionales, utilizamos la escala de Karnosfky. Para la medida de la CVRS empleamos el Cuestionario de Salud SF-36. Y para obtener el I CC se utilizó el Índice de Comorbilidad de Charlson.

\section{Recogida de datos}

La recogida de los datos se realizó en 2 días: un viernes por la mañana y por la tarde y un sábado con el mismo horario. Para ello, se explicó el objetivo del estudio a cada uno de los pacientes y se solicitó el consentimiento verbal para participar en el mismo. Se garantizó siempre la confidencialidad de los datos y el anonimato del paciente. La identidad de los pacientes solo era conocida por la enfermera responsable y el tutor.

La cumplimentación del cuestionario SF-36 se realizó durante la sesión de HD, de modo que el entrevistador formulaba la pregunta dando las posibles respuestas y el paciente contestaba a cada una de ellas. La escala de Karnofsky fue cumplimentada por la enfermera responsable de cada paciente. EI ICC se obtuvo mediante la revisión de los datos clínicos de cada paciente y fue incluida por el director del trabajo en la base datos en la que los pacientes no estaban identificados.

\section{Análisis estadístico}

Las variables cualitativas se representaron mediante distribución de frecuencias. Para las variables cuantitativas se utilizó la media \pm la desviación estándar de la media o la mediana y rango según siguieran o no una distribución normal. Para la relación entre variables se utilizó el coeficiente de correlación de Pearson. Los datos se analizaron con el programa SPSS 15.0 para Windows.

\section{Resultados}

La muestra de estudio estuvo formada por 43 pacientes con una edad media de $67.3 \pm 18.2$ años. La mediana del tiempo en HD fue 48 (4-360) y el tiempo medio de llegada al centro de diálisis de $21.6 \pm 13.6$ minutos. La mediana ICC fue de 7 (0-10) y la mediana de la escala de Karnosfky fue de 80 (30-100).
En la tabla 1 se representa la frecuencia y porcentaje de las variables sociodemográficas estudiadas.

Tabla 1.

\begin{tabular}{|c|c|}
\hline Sexo & \\
\hline Mujer & $14(32.6 \%)$ \\
\hline Hombre & $29(32.6 \%)$ \\
\hline \multicolumn{2}{|l|}{ Estado civil } \\
\hline Soltero/a & $9(20.9 \%)$ \\
\hline Casado/a & $27(62.8 \%)$ \\
\hline Viudo/a & $5(11.6 \%)$ \\
\hline Separado/a - Divorciado/a & $2(4.7 \%)$ \\
\hline \multicolumn{2}{|l|}{ Transporte utilizado } \\
\hline Taxi & $41(95.3 \%)$ \\
\hline Medio propio & $2(4.7 \%)$ \\
\hline \multicolumn{2}{|l|}{ Acceso vascular } \\
\hline FAVI & $33(76.7 \%)$ \\
\hline Catéter & $3(7,0 \%)$ \\
\hline Goretex & $3(7,0 \%)$ \\
\hline FAVI/Catéter & $3(7,0 \%)$ \\
\hline FAVI/Goretex & $1(2.3 \%)$ \\
\hline \multicolumn{2}{|l|}{ Situación laboral } \\
\hline Activo & $3(7,0 \%)$ \\
\hline Ama de casa & $9(20,9 \%)$ \\
\hline Paro & $1(2,3 \%)$ \\
\hline P. incapacidad laboral & $6(14,0 \%)$ \\
\hline P. jubilación & $20(46.5 \%)$ \\
\hline P. no contributiva & $1(2.3 \%)$ \\
\hline P. viudedad & $1(2.3 \%)$ \\
\hline Régimen especial & $2(4.7 \%)$ \\
\hline \multicolumn{2}{|l|}{ Composición hogar } \\
\hline Familia & $37(86,0 \%)$ \\
\hline Solo & $2(4,7 \%)$ \\
\hline Asistenta & $2(4,7 \%)$ \\
\hline Convento & $1(2,3 \%)$ \\
\hline Especial & $1(2,3 \%)$ \\
\hline
\end{tabular}

Respecto a la profesión, las mujeres eran, en su mayoría, amas de casa aunque algunas también trabajaban fuera del hogar familiar. Los hombres desempeñaban múltiples profesiones como ingeniero, constructor, transportista, profesor, etc.

En la tabla 2 se representan las categorías generales en que se clasifica el índice de Karnofsky junto con la frecuencia y porcentaje de cada grado. 
Tabla 2.

\begin{tabular}{|cccc}
\hline $\begin{array}{c}\text { Categorías } \\
\text { generales }\end{array}$ & Grado & Frecuencia & Porcentaje \\
\hline $\begin{array}{c}\text { Capaz de realizar actividades } \\
\text { normales, no requiere } \\
\text { cuidados especiales }\end{array}$ & 100 & 20 & 46.5 \\
\hline $\begin{array}{c}\text { Incapaz de trabajar, puede } \\
\text { vivir en casa y autocuidarse } \\
\text { con ayuda variable }\end{array}$ & 90 & 1 & 2.3 \\
\hline $\begin{array}{c}\text { Incapaz de autocuidarse. } \\
\text { Requiere cuidados especiales, } \\
\text { susceptible de hospitalización. } \\
\text { Probable avanne rápido de } \\
\text { enfermedad }\end{array}$ & 50 & 3 & 7.0 \\
\hline
\end{tabular}

Con respecto a la CVRS, las puntuaciones de las diferentes dimensiones se presentan en la tabla 3.

Tabla 3. Dimensiones SF-36

\begin{tabular}{ccccccccc}
\hline & $\begin{array}{c}\text { Función } \\
\text { física }\end{array}$ & $\begin{array}{c}\text { Salud } \\
\text { general }\end{array}$ & $\begin{array}{c}\text { Rol } \\
\text { físico }\end{array}$ & $\begin{array}{c}\text { Rol } \\
\text { emocional }\end{array}$ & $\begin{array}{c}\text { Dolor } \\
\text { corporal }\end{array}$ & Vitalidad & $\begin{array}{c}\text { Función } \\
\text { social }\end{array}$ & $\begin{array}{c}\text { Salud } \\
\text { mental }\end{array}$ \\
\hline Media & 49.65 & 40.46 & 50.58 & 81.78 & 55.69 & 51.45 & 58.43 & 69.65 \\
\hline Desv.Típ. & 30.06 & 22.08 & 34.47 & 28.30 & 31.96 & 31.57 & 36.05 & 22.81 \\
\hline
\end{tabular}

lud mental de la CVRS ( $r=0.36, p<0.05)$; del ICC con la escala de Karnofsky $(r=-0.54, p<0.01)$, la dimensión función física $(r=-0.43, p<0.01)$ y la dimensión función social de la CVRS $(r=-0.31, p<0.05)$.

Cuando relacionamos las categorías generales de la escala de Karnofsky (según grado de dependencia), encontramos una correlación estadísticamente significativa entre el ICC y la categoría 2 (Incapaz de trabajar) $(r=0.65, p<0.01)$ y con la categoría 3 (Incapaz de autocuidarse) del Karnofsky $(r=-0.62$, $p<0.01)$; del rol físico con la categoría 2 ( $r=0.34$, $\mathrm{p}<0.05)$; de la dimensión rol emocional con la categoría 1 (Capaz de realizar actividades normales $(r=-0.44, p<0.01)$ y con la categoría $2(r=0.441$, $\mathrm{p}<0.01)$; de la dimensión salud mental con la categoría $2(r=0.42$, $p<0.01)$ y con la categoría $3(r=$ $-0.34, p<0.05)$; y de la edad con la categoría $2(r=0.74, p<0.01)$ y con la categoría $3(r=-0.71, p<0,01)$.
Cuando comparamos nuestros resultados con los valores de referencia de la población general española ${ }^{16}$, podemos observar una peor CVRS, tal como puede apreciarse en la Figura 1.

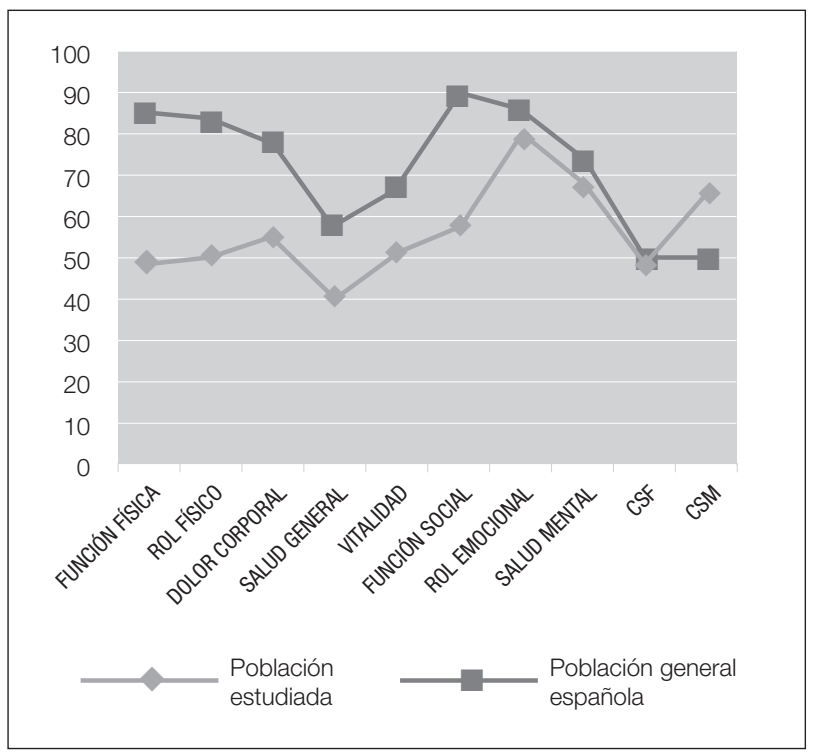

Figura 1. Valores medios de las diferentes dimensiones de la población estudiada y población general española.

Cuando se relacionaron las distintas variables, se encontró correlación estadísticamente significativa de la edad con la escala de Karnofsky ( $r=-0.58$, $p<0.01)$, el ICC $(r=0.81, p<0.01)$ y la dimensión sa-

\section{Discusión}

En la actualidad, la CVRS se considera un marcador clínico más en las enfermedades de tipo crónico como la IRC, pues el paciente ha de aprender a convivir con las limitaciones que ocasiona la propia enfermedad y la aplicación de las distintas formas de tratamiento, que modifican su estilo de vida en mayor o menor medida $^{17}$.

Nuestro objetivo era conocer la CVRS de los pacientes en HD del centro periférico de diálisis de la provincia de Córdoba. Nuestros resultados ponen de manifiesto una peor CVRS en los pacientes en HD con respecto a la población general, con bajas puntuaciones en todas las dimensiones del SF-36. Entre ellas, la dimensión rol físico es la más afectada, seguida de la función social y función física, respectivamente; y las menos afectadas son las dimensiones salud mental y rol emocional. Esto es lógico, debido a elevada edad, comorbilidad y nivel de dependencia, que condiciona que los pacientes tengan dificultades en sus actividades diarias y limitaciones importantes en su vida social y laboral, de los que aún están en edad laboral ${ }^{18}$.

Trabajos previos que han utilizado el mismo instrumento de medida han evidenciado, también, un deterioro en todas y cada una de las dimensiones de la CVRS de los pacientes en programas de HD respecto a la población general ${ }^{19}$, coincidiendo con nuestra 
nuestros resultados en que la función física es de las más afectadas y que la salud mental es una de las menos afectadas ${ }^{20}$. Un gran estudio multicéntrico, realizado por la National Kidney Foundation en 1000 pacientes en HD utilizando el SF-36, comparándolos con una población sana de 2474 sujetos, reveló que la función física es la dimensión más afectada en los pacientes en HD, mientras que la dimensión mental es la más cercana a la normalidad ${ }^{18}$.

En otro estudio realizado en 2006, la dimensión función física y rol físico también son de las más afectadas, junto con rol emocional; mientras que la función social es una de las dimensiones menos afectadas junto con salud general y vitalidad 21,22 .

A pesar de que todas las dimensiones están por debajo de los valores de referencia, nos ha llamado la atención la puntuación obtenida en la dimensión rol emocional, pues esperábamos obtener una puntuación mucho más baja, como ocurre en otros estudios sobre calidad de vida ${ }^{12}$. Esto parece indicar que los pacientes en HD presentan una buena adaptación psicológica a todos los cambios que produce la enfermedad y el tratamiento en sus vidas.

Nuestros resultados también muestran la influencia de la comorbilidad sobre la CVRS, principalmente en las dimensiones rol físico y función social. Esto confirma el hallazgo de otros autores de que, en pacientes tratados con HD, mayor comorbilidad se asocia a peor calidad de vida. El conocimiento de la comorbilidad en cualquier unidad de diálisis parece un objetivo importante, para prevenir en todo lo posible la aparición de patologías añadidas, que además de mejorar la supervivencia, permitiría mejorar la percepción que el paciente tiene de su salud ${ }^{13}$.

Respecto al género, son numerosos los estudios que asocian el sexo femenino con una peor CVRS en los pacientes en terapia renal sustitutiva ${ }^{14}$. Esto podría explicarse por el hecho de que la mujer soporta mayor carga social que el hombre, hecho que se intensifica con la edad ya que confluyen varias generaciones al cuidado de ella. Esta correlación se ha observado en otros estudios tanto en pacientes en diálisis como en la población general ${ }^{23,24}$. Sin embargo, no ocurre igual en nuestro estudio, donde no existe correlación estadísticamente significativa del sexo con ninguna de las dimensiones del SF-36 ni con las variables estudiadas.

Acerca de la edad, se ha mostrado como uno de los factores más influyente en la CVRS ${ }^{25,26}$, igual que ocurre en los pacientes de nuestra muestra. Sin em- bargo, esta influencia es sobre la dimensión salud mental y no sobre la función física como esperábamos. Sería interesante realizar una investigación posterior clasificando a los pacientes por grupos de edad para comprobar que grupo es el que obtiene mejores resultados en la CVRS como ya se hiciera en otros estudios, donde se muestra que los pacientes de mayor edad son los que presenta peor CVRS 27,28 . Esto es lógico, pues un aspecto común dentro del envejecimiento lo constituye el hecho de que, la edad no hace más que acentuar las carencias de cada persona, de modo que en muchos casos se necesita ayuda de terceras personas, tanto en el ámbito físico (sobre todo para las actividades básicas de la vida diaria), psicológico (especialmente emocional y afectivo), socioeconómico (entorno familiar, de la comunidad e instituciones).

Referente al grado de dependencia, en nuestros pacientes se ha encontrado relación entre los resultados de esta escala de Karnofsky con la CVRS, con la comorbilidad y la edad; es decir los pacientes más dependientes son los que tienen más edad, presentan peor CVRS y tienen más comorbilidad, similar a 10 encontrado por otros autores ${ }^{29}$.

La principal limitación de este estudio fue el muestreo, pues solo tuvimos acceso a la población de pacientes en HD, del Centro Periférico de Diálisis, cuyos pacientes tienen la particularidad de que son pacientes ambulatorios, y de ahí se obtuvo la muestra de acuerdo a los criterios de inclusión/exclusión.

Como principales conclusiones, podemos decir que los pacientes estudiados, presentan peor CVRS, con bajas puntuaciones en todas las dimensiones del SF-36 en comparación con los valores de referencia de la población general española. Entre ellas, la dimensión rol físico es la más afectada, seguida de la función social y función física, respectivamente; y las menos afectadas son las dimensiones salud mental y rol emocional. Esta peor CVRS se asocia con mayor edad, comorbilidad y dependencia. Con respecto a la dependencia, el $44 \%$ de los pacientes presentan algún grado de dependencia relacionada fundamentalmente con la edad y la comorbilidad. Por otra parte, las medidas de la CVRS son útiles para mejorar la comunicación entre el paciente y el personal sanitario, de tal forma que el primero percibe el interés de los segundos por sus expectativas, sensaciones y preferencias. Los profesionales de la salud deberían estar familiarizados con la aplicación de las herramientas de evaluación de la calidad de vida, que puede ser valioso en la ayuda global de estos pacientes, incluso en las etapas tempranas de 
la enfermedad, y permitir oportunas intervenciones en el curso de la enfermedad.

\section{Agradecimientos}

A todo el personal de enfermería del Centro Periférico de Diálisis del Hospital Universitario Reina Sofía de Córdoba. A los pacientes por su paciencia y colaboración., sin cuya participación no hubiera sido posible la realización de este Trabajo Fin de Grado.

A Antonio Pesquero Villarejo, por su continuo apoyo y colaboración.

Recibido: 10 Agosto 2014

Revisado: 20 Agosto 2014

Modificado: 22 Agosto 2014

Aceptado: 22 Agosto 2014

\section{Bibliografía}

1. Arenas M D, Álvarez-Ude F, Angoso M, Berdud I, Antolín A, Lacueva J, García Marcos S, Fernández A, Gil M T, Soriano A. Valoración del grado de dependencia funcional de los pacientes en hemodiálisis (HD): estudio multicéntrico. Nefrología. 2006; 26 (5): 600-608.

2. Carrillo Algarra A J, Laza Vásquez C, Molina Jerena J A. Estudio documental (2006-2013) sobre el autocuidado en el día a día del paciente con enfermedad renal crónica. Enferm Nefrol 2013; 16 (3): 185-192.

3. Menéndez J, Guevara A, Arcia N, León Díaz E M, Marín C, Alfonso J C. Enfermedades crónicas y limitación funcional en adultos mayores: estudio comparativo en siete ciudades de América Latina y el Caribe. Rev Panam Salud Pública 2005: 17(5/6): 353-361.

4. Cristóvão A F. Dificultades y estrategias en el manejo del régimen terapéutico en el paciente renal crónico en hemodiálisis. Enferm Nefrol. 2013; 16 (4): 247-255.

5. González Prieto V C, Lobo Llorente N. Calidad de vida en los pacientes con insuficiencia renal crónica terminal en tratamiento sustitutivo de hemodiálisis. Aproximación a un proyecto integral de apoyo. Rev Soc Esp Enferm Nefrol. 2001; 4 (14): 6-12.

6. Rodríguez Calero M A, Hernández Sánchez D, Gutiérrez Navarro M J, Juan Amer F, Calls Ginesta J. Evaluación del dolor crónico en una población de pacientes hemodializados. Rev Soc Esp Enferm Nefrol. 2007; 10 (2): 137-143.

7. Cobo Sánchez $J$ L, Pelayo Alonso R, Ibarguren Rodríguez E, Aja Crespo A, Saenz de Buruaga Perea $A$, Incera Setién $M E$ et al. Factores sociológicos y calidad de vida relacionada con la salud en pacientes en hemodiálisis. Rev Soc Esp Enferm Nefrol. 2011; 14 (2): 98-104.

8. Andreu Periz L, Moreno Arroyo C, Julve Ibañéz $M$. Valoración de pacientes tratados con hemodiálisis según la ley de promoción de la autonomía personal y atención a las personas en situación de dependencia. Rev Soc Esp Enferm Nefrol. 2010; 13 (4): 252-257.

9. Cobo Sánchez $J$ L, Pelayo Alonso $R$, Sánchez Cano M S, Alonso Nates R, Menezo Viadero R, Gándara Revuelta et al. Repercusión del grado de dependencia de los pacientes en hemodiálisis sobre la carga de trabajo de enfermería. Rev Soc Esp Enferm Nefrol. 2008; 11 (1): 6-11.

10. Andreu Periz L, Puig LLobet M, Cases Amenós A. Grado de dependencia de las personas sometidas a hemodiálisis en Cataluña y evolución de su mortalidad. Nefrología. 2012; 32(5):613-21.

11. Álvarez F, Fernández M, Vázquez A, Mon C, Sánchez R, Rebollo P. Síntomas físicos y trastornos emocionales en pacientes en programa de hemodiálisis periódicas. Nefrología. 2001; 21 (2): 191-199.

12. Seguí Gomà $A$, Amador Peris $P$, Ramos Alcario A B. Calidad de vida en pacientes con insuficiencia renal crónica en tratamiento con diálisis. Rev Soc Esp Enferm Nefrol. 2010; 13 (3): 155-160.

13. Rebollo P, Bobes J, González M P, Saiz P, Ortega $F$. Factores asociados a la calidad de vida relacionada con la salud (CVRS) de los pacientes en terapia renal sustitutiva (TRS). Nefrología. 2000; 20 (2): 171-181.

14. Gil Cunqueiro J M, García Cortés M J, Foronda J, Borrego J F, Sánchez Perales M C, Pérez del Barrio $P$ et al. Calidad de vida relacionada con la salud en pacientes ancianos en hemodiálisis. Nefrología. 2003; 23 (6): 528-537. 
15. Ortega N, Martínez M. Bienestar psicológico como factor de dependencia en hemodiálisis. REV Enferm IMSS. 2002; 10 (1): 17- 20.

16. Iraurgi Castillo I, Póo M, Márkez Alonso I. Valoración del índice de salud sf-36 aplicado a usuarios de programas de metadona. Valores de referencia para la comunidad autónoma vasca. Rev Esp Salud Pública. 2004; 78 (5): 609-621.

17. Jofre R, López Gómez JM, Valderrábano F. Quality of life for patient groups. Kidney Int 2000; 57 (Suppl 74): 121-30.

18. Jofré R. Factores que afectan a la calidad de vida en pacientes en prediálisis, diálisis y trasplante renal. Nefrología. 1999; 19 (Supl. 1): 84-90.

19. Carmichael P, Popoola J, John I, Stevens PE, Carmichael AR. Assessment of quality of life in a single centre dialysis population using the KDQOL-SF questionnaire. Qual Life Res. 2000; 9 (2): 195-205.

20. Morales Jaimes R, Salazar Martínez E, Flores Villegas F J, Bochicchio Riccardellic T, López-Caudana. Calidad de vida relacionada con la salud en los pacientes con tratamiento sustitutivo renal: el papel de la depresión. Gac Méd Méx Vol. 144 No. 2, 2008, 91-98.

21. Contreras F, Espinosa J C, Esguerra G A, Gutiérrez C, Fajardo L. Calidad de vida y adhesión al tratamiento en pacientes con insuficiencia renal crónica en tratamiento de hemodiálisis. Univ. Psychol. 2006; 5 (3): 487-499.

22. Fujisawa M, Ichikawa $Y$, Yoshiva $K$, Isotani $S$, Higuchi $A$, Nagano $S$ et al. Assessment of HealthRelated quality of life in renal transplant and hemodialysis patients using the SF-36 health survey. Urology. 2000; 56 (2): 201-206.
23. Carmichael P, Popoola J, John I, Stevens PE, Sarmichael AR. Assessment of quality of life in a single centre of dialysis population using the KDQOLSFTM questionnaire. Qual Life Res. 2000; 9 (2): 195-205.

24. Mittal SK, Ahern L, Flaster E, Maesaka JK, Fishbane $\mathrm{S}$. Selfassessed physical and mental function of haemodialysis patients. Nephrol Dial Transplant. 2001; 16 (7): 1387-1394, 2001.

25. Cruz M C, Andrade C, Urrutia M, Draibe $S$, Nogueira Martins L A, de Castro Cintra Sesso R. Quality of life in patients with chronic kidney disease. Clinics. 2011; 66 (6): 991-995.

26. Álvarez-Ude F, Vicente E, Badía X. La medida de la calidad de vida relacionada con la salud en los pacientes en programa de hemodiálisis y diálisis peritoneal continua ambulatoria de Segovia. Nefrología. 1995; 15 (6): 572-580.

27. Rebollo P, Ortega F, Baltar JM, Álvarez-Ude F, Álvarez Navascués R, Álvarez Grande J. Is de the loss of health related quality of life during renal replacement therapy lower in elderly patients than in younger patients? Nephrology Dialysis Transplantation; 2001, 16(8): 1675-1680.

28. Álvarez F. Factores asociados al estado de salud percibido (calidad de vida relacionada con la salud) de los pacientes en hemodiálisis crónica. Revista de la Sociedad Española de Enfermería Nefrológica. 2001; 14: 64-68.

29. Arenas M D, Moreno E, Reig A, Millán I, Egea J $J$, Amoedo $M L$ et al. Evaluación de la calidad de vida relacionada con la salud mediante las láminas Coop-Wonca en una población de hemodiálisis. Nefrología. 2004; 24 (5): 470-479. 